\title{
Effect of Valeriana fauriei extract on the offspring of adult rats exposed to prenatal stress
}

\author{
HWAYOUNG LEE ${ }^{1}$, HANSOL WON ${ }^{1}$, JIYUN IM ${ }^{1}$, YOUNG OCK KIM ${ }^{2}$, SANGHYUN LEE $^{3}$, \\ IK-HYUN CHO $^{4}$, HYUNG-KI KIM ${ }^{1}$, JUN-TACK KWON ${ }^{1}$ and HAK-JAE KIM ${ }^{1,5}$
}

\author{
${ }^{1}$ Department of Clinical Pharmacology, College of Medicine, Soonchunhyang University, Cheonan; \\ ${ }^{2}$ Development of Ginseng and Medical Plants Research Institute, Rural Administration, Eumseong; \\ ${ }^{3}$ Department of Integrative Plant Science, Chung-Ang University, Anseong; ${ }^{4}$ Department of Convergence Medical Science \\ and Brain Korea 21 Plus Program, Institute of Korean Medicine, College of Oriental Medicine, Kyung Hee University, Seoul; \\ ${ }^{5}$ Soonchunhyang Medical Research Institute, College of Medicine, Soonchunhyang University, Cheonan, Republic of Korea
}

Received August 14, 2015; Accepted April 20, 2016

DOI: $10.3892 / \mathrm{ijmm} .2016 .2589$

\begin{abstract}
Exposing a pregnant female to stress is a risk factor for the development of psychiatric disorders in the offspring. In the present study, we examined the effects of an extract of Valeriana fauriei (VF) root $(100 \mathrm{mg} / \mathrm{kg} /$ day, administered on postnatal days 35-56) on behavioral patterns as well as protein expression in the prefrontal cortex of the offspring of prenatallystressed rats. Modified behavioral tests, including the forced swim test, the open field test, a social interaction test and the prepulse inhibition test were performed and many of the parameters were found to decrease in the offspring of the rats exposed to PNS compared with the offspring of the non-stressed rats. Western blot and immunohistochemical analyses of the prefrontal cortex revealed that the downregulation of several neurodevelopmental proteins in the offspring of rats dams exposed to PNS was reversed after treatment with VF extract. These findings demonstrate that the downregulation of several proteins in the prefrontal cortex of the offspring of prenatally-stressed rats may be associated with subsequent behavioral changes, and that these phenomena recovered following VF treatment. Our results suggest that VF decreases the incidence of prenatal stress related-psychiatric disorders, such as depression and schizophrenia.
\end{abstract}

\section{Introduction}

Valeriana is the main genus in the family Valerianaceae, and valerian root extracts have been used as a traditional herbal medicine for centuries (1). The genus Valeriana contains $>250$ species and many subspecies (2). Valeriana fauriei Briq. (VF)

Correspondence to: Professor Hak-Jae Kim, Department of Clinical Pharmacology, College of Medicine, Soonchunhyang University, 31 Soonchunyang 6-gil, bongmengdong, Dongnamgu, Cheonan 330-721, Republic of Korea

E-mail: hak3962@sch.ac.kr

Key words: prenatal stress, animal behavior, Valeriana fauriei, psychiatric disorder has been used to treat humans for hundreds of years (3). In certain countries, it is primarily sold as a sleeping aid, and in Europe it is used to treat restlessness, tremors and anxiety (4-8). Valeriana officinalis has been used as a sedative and to treat anxiety and sleep disorders $(9,10)$. Various effects of $V$. officinalis have been reported; it has been suggested that Valeriana exerts its effects through gamma-aminobutyric acid (GABA) ergic mechanisms (11). In a previous study, V. officinalis exerted antioxidant effects and decreased lipid peroxidation induced by quinolinic acid (12). In addition, V. officinalis has been reported to exert neuroprotective effects in several neurodegenerative diseases, such as Parkinson's and Alzheimer's disease (13-15).

Prenatal stress (PNS) during the critical period of fetal brain development is an important environmental risk factor for the development of human psychiatric disorders, such as schizophrenia, in the adult offspring, and the second trimester of pregnancy in humans seems to be the most vulnerable period for insult (16-21).

Additionally, previous studies have demonstrated that PNS elevates glucocorticoids during gestation and is associated with biochemical, physiological and behavioral changes in the offspring, including reduced birth weight, cardiovascular and neuroendocrinological abnormalities, attention dysfunction, enhanced anxiety-related behaviors and cognitive deficits (22-31). Thus, the pregnant rats in the present study were exposed to stressful manipulations during the third week of pregnancy, which is similar to the second trimester of human gestation (28-30). Previous studies have shown that PNS decreases dendritic length, spine density, the number of neurons, and diminishes the number of hippocampal synapses as compared with non-stressed (NS) controls $(32,33)$. PNS also causes various changes in gene expression, including the expression of genes associated with neural development, cell differentiation, and neurotransmitter function in the brains of rats $(27,34,35)$.

To the best of our knowledge, no previous studies have examined the effects of VF on PNS or neurodegeneration. In the present study, behavioral patterns and changes in protein levels were examined in the prefrontal cortex of the offspring of rats exposed to PNS, and we subsequently determined whether the changes caused by PNS were affected by treatment with VF. 


\section{Materials and methods}

Preparation of VF extracts and administration to rats. VF root extract was purchased from Yunpung (Chungbuk, Korea), and the specimens were identified taxonomically by an Oriental medicine physician at the National Institute of Horticultural and Herbal Science [Rural Development Administration (RDA); Wanju, Jeonbuk, Korea]. The voucher specimen (HPR-207) was deposited in the herbarium of the Herbal Crop Research Institute (Eumseong, Korea).

Drugs and animals. VF was dissolved in water, and the drug was administered on postnatal day 35 for 3 weeks until postnatal day 56 , as previously described (31). It was provided to the rats in regular drinking bottles $(100 \mathrm{mg} / \mathrm{kg} / \mathrm{day})$ to avoid preadolescent stress exposure resulting from the administration of repeated injections.

Prenatal stress procedures. Prenatal stress procedures. Pregnant Sprague-Dawley rats ( $n=6$ in each group) were purchased from Central Lab Animal, Inc. (Seoul,Korea) and arrived at the animal facility on day 7 of gestation. The rats were housed under standard conditions with a 12/12-h light/ dark cycle (lights on at 06:30) with free access to food and water. All animal procedures were performed in accordance with the Guidelines for the Care and Use of Laboratory Animals of the US National Institutes of Health. All experimental procedures were reviewed and approved by the institutional Review Board for Animal Welfare at Soonchunhyang University. The rat model of PNS was established as described in our previous studies $(36,37)$. Briefly, PNS exposure was initiated on day 14 of gestation and continued until day 21, and consisted of: i) $1 \mathrm{~h}$ restraint in a well-ventilated, cylindrical Plexiglas restraint device (Braintree Scientific, Inc. Braintree, MA, USA), ii) $6 \mathrm{~h}$ of exposure to a cold environment $\left(4^{\circ} \mathrm{C}\right)$, iii) overnight fasting, iv) $15 \mathrm{~min}$ of swimming stress in room-temperature water, v) reversal of the light-dark cycle, and/or vi) social stress induced by overcrowded housing conditions during the dark phase $(28,29)$. Pregnant dams used as controls remained in the animal housing room during gestational days 14-21 and were exposed to only normal animal husbandry procedures.

All groups contained litters of between 8 and 15 pups with similar numbers of males and females, extremely large or small litters being eliminated. The offspring were weaned 21 days after birth and group-housed. The male offspring were selected and used for further experiments. Thus, 3 experimental groups were tested as adults: the 'control' ( $n=16$ male offspring) group were offspring of unstressed mothers; the prenatal stress group 'PNS' ( $n=16$ male offspring) were offspring of mothers subjected to stress before parturition; and the VF group, VF administration in a group subjected to prenatal stress $(n=16$ male offspring).

Behavioral tests. Modified behavioral tests, including the forced swim test (FST), the open field test (OFT), a social interaction test (SIT), and the prepulse inhibition (PPI) test were performed as previously described (28,37-40).

PPI test. Briefly, an automated startle reflex system (SR-Lab, San Diego Instruments, San Diego, CA, USA) was used to measure PPI. The system consisted of a startle chamber housed in a sound-attenuated isolation cabinet equipped with an internal fan and light. A cylindrical, transparent, acrylic holding apparatus resting on a four-pegged platform within the isolation chamber was used to hold each subject throughout the testin $g$ session (subject age, 56 days). Background noise and acoustic stimuli were controlled via the SR Lab microcomputer and interface assembly, and were delivered through a speaker mounted above the cylindrical holding apparatus. All test chambers were located in a sound-attenuated experimental room to minimize external noise, as previously described (41). Background noise of $70 \mathrm{~dB}$ was present throughout the test session. After a 5-min acclimation period to the background noise, each subject was presented with a series of 60 acoustic stimuli trials. The trials were presented in pseudorandom order, namely the individual startle trials (single acoustic stimulus delivered at $120 \mathrm{~dB}$ for $40 \mathrm{msec}$ ), the prepulse stimulus trials (a single prepulse stimulus presented at $15 \mathrm{~dB}$ above background for $20 \mathrm{msec}$ ), the nonstimulus trials (not following any noise), and the prepulse stimulus trials with acoustic stimuli (a single prepulse stimulus presented at $15 \mathrm{~dB}$ above background, followed $20 \mathrm{msec}$ later by a startle stimulus presented at $120 \mathrm{~dB}$ for $40 \mathrm{msec}$ ). The intertrial interval was $15 \mathrm{msec}$, and each session lasted $22 \mathrm{~min}$. The holding chambers were cleaned with $75 \%$ ethanol between each test session. Prepulse inhibition was presented as the percentage decrease in startle amplitude as a function of the magnitude of the prepulse stimulus using the following formula: percentage decrease $=100 x$ [(acoustic stimuli trial $)-($ the prepulse stimulus trials + acoustic stimuli)] (42).

FST. The FST was performed (subject age, 57 days) as previously described (37-39). The rats were lowered individually into a cylinder filled with fresh warm tap water $\left(25 \pm 2^{\circ} \mathrm{C}\right)$. The rat was removed after $15 \mathrm{~min}$ and wiped with a clean towel to remove excess water before being returned to its home cage. Each rat was placed in the cylinder again for $5 \mathrm{~min}$ the following day, and swimming, climbing and immobility behaviors were recorded with a video camera (Samsung HMX-T10) and by an observer with a stopwatch.

OFT. The OFT was performed in order to assess exploratory activity and reactivity to a novel environment. The subjects were removed from their home cage on the day of the test (subject age, 59 days) and placed individually in an open-field start box for $5 \mathrm{~min}$. The apparatus was constructed from black polygal panels (Dowin Polychem Co., Ltd., Seoul, Korea), and no background noise was provided. The experimenter exited the room, and the behavior of the subject was recorded, as previously described (37-39).

SIT. The SIT was adapted from previous studies $(28,39,40)$ (subject age, 58 days). The social interaction partners were same-sex siblings that resided in the same cage after weaning and were of approximately equal body weight. Each session lasted $20 \mathrm{~min}$ and was scored in terms of total duration of social play and the number and type of interactions.

Measurement of corticosterone. Following the behavior tests, the adult (59 days of age) male offspring from each group were moved to the laboratory. At approximately 16:00 on the last behavior test day, the experimental subjects were placed in cylindrical plastic restraint tubes for $60 \mathrm{~min}$ ( $n=5-6$ animals/group). The restraint stressed male offspring ( $n=5-6$ animals/group) were sacrificed by decapitation. Some 
of the rats were anesthetized with ethyl ether and perfused with $4 \%$ paraformaldehyde. ( $n=5-6$ animals/group). Trunk blood was collected immediately in plastic tubes. The blood was centrifuged at 13,000 rpm, and the serum was placed in a fresh tube. The brains were removed rapidly from the skull and the prefrontal cortex and hippocampus were separated, placed in a fresh tube, and frozen in liquid nitrogen. The brain tissue and serum were stored at $-80^{\circ} \mathrm{C}$ until use. Serum corticosterone levels were determined by immunoassay using the Rat Cortisol ELISA kit purchased from MyBioSource (cat no. MBS023335; San Diego, CA, USA). Assays were conducted according to the manufacturer's instructions, as previously described (43).

Western blot analysis. Prefrontal cortical tissues were lysed in RIPA buffer containing protease inhibitors and centrifuged at $14,000 \mathrm{rpm}$ for $10 \mathrm{~min}$ at $4^{\circ} \mathrm{C}$. To detect dihydropyrimidinase-like 2 (Dpys12) and the neurofilament protein, $80 \mu \mathrm{g}$ lysed protein was subjected to 10 and $12 \%$ sodium dodecyl sulfate-polyacrylamide gel electrophoresis and transferred to a polyvinylidene difluoride membrane (Millipore, Milford, MA, USA). After blocking with 5\% skim milk, the membranes were probed with anti-Dpys12 (1:1,000; \#9393, Cell Signaling Technology, Danvers, MA, USA), anti-LIM and SH3 protein 1 (Lasp1; 1:2,000; MAB8991, Millipore), anti-neurofilament M (Nefm; 1:1,000; \#2838, Cell Signaling Technology), anti-PSD95 [discs, large homolog 4 (Dlg4); 1:1,000; \#3450, Cell Signaling Technology] or anti- $\beta$-actin (Actb; $1: 1,000$; sc-81178, Santa Cruz Biotechnology, Inc., CA, USA) antibodies overnight at $4^{\circ} \mathrm{C}$ and then with peroxidase-conjugated secondary antibody (1:10,000; N4142, Sigma, St. Louis, MO, USA) for $1 \mathrm{~h}$ at room temperature. Immunoreactive bands were detected using an enhanced chemiluminescence kit (Elpis-Biotech, Inc., Daejeon, Korea), and quantitative measurements of Dpys12, Lasp1, Nefm, Dlg4 and Actb proteins were calculated using ImageJ software.

Immunohistochemistry. The rats were anesthetized with ethyl ether and perfused with $4 \%$ paraformaldehyde. The fixed brains were removed, frozen and cut into $30-\mu \mathrm{m}$ sections. Frozen sections from the prefrontal cortex were blocked with normal horse serum, incubated with anti-Dpysl2 (1:1,000; HPA002381, Atlas Antibodies AB, Stockholm, Sweden), Nefm (1:100; \#2838, Cell Signaling Technology) and anti-NeuN (1:100; MAB377, Millipore) and then incubated with Cy3-conjugated anti-rabbit and mouse secondary antibodies (1:500 and 1:800; 715-545-151, 111-165-003, Jackson ImmunoResearch Laboratories, Inc., West Grove, PA, USA). Nuclei staining was carried out using 4',6-diamidino-2-phenylindole (DAPI) staining (Sigma). Fluorescent images were subsequently captured using a confocal laser scanning microscope (FV10-ASW; Olympus, Tokyo, Japan), and the images were quantified with Image J software according to a protocol described previously with minor modifications (44).

Statistical analysis. All data are expressed as the means \pm standard deviation and/or standard error of the means and compared using the Student's t-test. All statistical analyses were performed using IBM SPSS Statistics 19 software (SPSS Inc., Chicago, IL, USA). A p-value $<0.05$ was considered to indicate a statistically significant difference.



Figure 1. Prepulse inhibition (PPI) in adult male rats. This was measured on postnatal day 56 in adult male rats exposed to stress during gestation or those derived from litters that were not stressed during gestation. Eight male rats were included in each group. PPI is presented as the percentage decrease in startle amplitude as a function of the magnitude of the prepulse stimulus using the following formula: percentage decrease $=100 \times[$ (acoustic stimuli trial $)-($ the prepulse stimulus trials + acoustic stimuli)]. Each bar represents the mean of eight male rats $( \pm$ standard deviation), ${ }^{*} \mathrm{p}<0.05$, NS versus PNS group; ${ }^{* *} \mathrm{p}<0.05$, PNS versus VF group; NS, offspring of non-stressed rats; PNS, offspring of prenatally-stressed rats; VF, Valeriana fauriei-treated offspring.

\section{Results}

We established a rat model of variable and unpredictable PNS in order to evaluate the extent to which VF treatment altered behavioral patterns and protein expression in the prefrontal cortex. Thus, we aimed to examine the effects of VF on the pathophysiology of stress-induced psychiatric disorders caused by maternal stress during E14 to E21 of pregnancy.

PPI test. Prenatal stress exposure during the third week of gestation significantly affected sensorimotor gating. Significant prepulse facilitation was detected in offspring of prenatally-stressed rats (PNS group) at lower prepulse stimulus intensities, whereas no change in the startle response was detected in the offspring of NS rats (NS group) (Fig. 1). In order to examine the effect of VF on sensorimotor gating function, we measured the PPI level using the acoustic startle response test in the NS, PNS and VF groups. The results revealed a significantly different effect on PPI between the NS and PNS groups. The results indicate that PNS significantly altered the PPI level at prepulse stimulus levels of 1 and $10 \mathrm{~dB}$ above background between the PNS and NS offspring $(\mathrm{p}<0.05)$ but not at $50 \mathrm{~dB}$ above background ( $>00.05)$. VF treatment affected the PPI level at 71 and $80 \mathrm{~dB}$ pulse levels ( $\mathrm{p}>0.05)$; however, VF treatment decreased the PPI level at $120 \mathrm{~dB}$ pulse level $(\mathrm{p}<0.001)$.

FST. We noted significant differences among the NS, PNS, and VF-treated groups in the FST (Table I). The offspring of rats exposed to PNS exhibited decreased swimming ability and increased immobility compared with the offspring of NS rats ( $<<0.05$, Fig. 2). The changed behavior recovered after VF treatment ( $<<0.05$, Fig. 2).

OFT. The offspring from the NS and PNS groups were tested with the OFT for $20 \mathrm{~min}$. The PNS group had a significantly decreased number of central entries and line crossings as well as a decreased number and duration of rearing behaviors; these scores recovered to normal levels following VF treatment 
Table I. Behavior of the offspring of prenatally-stressed and non-stressed rats as well as of the offspring of prenatally-stressed rats treated with Valeriana fauriei extract in a forced swim test.

\begin{tabular}{lrrr}
\hline Behavior & NS (sec) & PNS (sec) & VF (sec) \\
\hline Swimming $^{\mathrm{a}, \mathrm{b}}$ & $31.88 \pm 3.53$ & $19.25 \pm 1.44$ & $26.88 \pm 1.83$ \\
Climbing $^{\mathrm{b}}$ & $27.88 \pm 3.44$ & $26.38 \pm 1.89$ & $32.50 \pm 1.87$ \\
Immobility $^{\mathrm{a}, \mathrm{b}}$ & $0.25 \pm 0.16$ & $14.00 \pm 1.65$ & $0.63 \pm 0.32$
\end{tabular}

Data are presented as the means \pm SEM; NS, non-stressed group; PNS, prenatally-stressed group; VF, oral administration of Valeriana fauriei

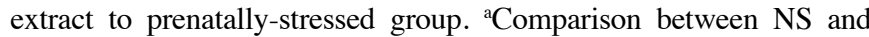
PNS, p-value $<0.05$; ${ }^{\mathrm{b}}$ comparison between PNS and VF, p-value $<0.05$.

Table II. Behavior of the offspring of prenatally-stressed and non-stressed rats as well as of the offspring of prenatally-stressed rats treated with Valeriana fauriei extract in an open field test.

\begin{tabular}{lccc}
\hline Behavior & NS & PNS & VF \\
\hline Central $^{\mathrm{a}, \mathrm{b}}$ & $6.14 \pm 0.94$ & $1.14 \pm 0.46$ & $9.43 \pm 2.29$ \\
Line crossing $^{\mathrm{a}, \mathrm{b}}$ & $3.43 \pm 0.53$ & $0.00 \pm 0.00$ & $3.42 \pm 0.75$ \\
Run(n) & $0.14 \pm 0.14$ & $0.00 \pm 0.00$ & $0.00 \pm 0.00$ \\
Run(s) & $0.14 \pm 0.14$ & $0.00 \pm 0.00$ & $0.00 \pm 0.00$ \\
Rear(n) ${ }^{\mathrm{a}, \mathrm{b}}$ & $72.71 \pm 5.83$ & $18.43 \pm 3.08$ & $68.29 \pm 5.27$ \\
Rear(s) & $296.14 \pm 29.18$ & $78.85 \pm 16.88$ & $338.43 \pm 32.49$ \\
Grooming(n) & $9.57 \pm 1.57$ & $21.00 \pm 3.29$ & $14.71 \pm 1.15$ \\
Grooming(s) & $121.57 \pm 18.19$ & $262.86 \pm 39.75$ & $189.86 \pm 33.59$ \\
Cage(n) & $107.43 \pm 6.40$ & $78.00 \pm 33.01$ & $78.57 \pm 5.43$ \\
Cage(s) & $313.29 \pm 17.42$ & $206.86 \pm 15.09$ & $241.29 \pm 21.98$ \\
Immobile(n) & $0.00 \pm 0.00$ & $45.86 \pm 6.04$ & $0.00 \pm 0.00$ \\
Immobile(s) $)^{\mathrm{a}, \mathrm{b}}$ & $0.00 \pm 0.00$ & $248.00 \pm 53.46$ & $0.00 \pm 0.00$ \\
\hline
\end{tabular}

Data are presented as the means \pm SEM. 'Central' denotes central boxes entered and 'Cage' denotes cage sniffing. $n$, number of times behavior was noted; s, duration measured in seconds; NS, non-stressed group; PNS, prenatally-stressed group; VF, oral administration of Valeriana fauriei extract to the prenatally-stressed group; ${ }^{a}$ Comparison between NS and PNS, $\mathrm{p}<0.05$; ${ }^{\mathrm{b}}$ comparison between PNS and VF, $\mathrm{p}<0.05$.

$(\mathrm{p}<0.05$, Table II). In the PNS group, we noted a significant increase in the number and duration of grooming episodes and immobility behaviors; these scores also recovered to the normal level following VF treatment ( $\mathrm{p}<0.001$, Table II).

SIT. Certain behavior scores measured during the SIT decreased significantly in the PNS group, namely, sniffing, following and grooming the partner. These decreased scores increased towards normal levels following VF treatment $(\mathrm{p}<0.05$, Table III). We also noted that certain scores increased in the PNS group: fighting, aggressive grooming (the partner) and biting. These increased scores also returned to normal levels following VF treatment.

Corticosterone. In the PNS group, we noted increased stress-induced corticosterone levels ( $p<0.05$, Fig. 3) compared with the NS group. The corticosterone level in the VF treatment
Table III. Behavior of the offspring of prenatally-stressed and non-stressed rats as well as of the offspring of prenatally-stressed rats treated with Valeriana fauriei extract in a social interaction test.

\begin{tabular}{|c|c|c|c|}
\hline Behavior & NS & PNS & VF \\
\hline $\operatorname{Sniffing}(n)^{a, b}$ & $84.57 \pm 8.20$ & $42.29 \pm 4.03$ & $76.57 \pm 5.22$ \\
\hline $\operatorname{Sniffing}(s)^{a, b}$ & $176.71 \pm 6.28$ & $57.71 \pm 7.92$ & $145.57 \pm 11.40$ \\
\hline Following $(\mathrm{n})^{\mathrm{a}, \mathrm{b}}$ & $22.00 \pm 5.42$ & $7.57 \pm 1.21$ & $17.71 \pm 3.29$ \\
\hline Following $(\mathrm{s})^{\mathrm{a}, \mathrm{b}}$ & $82.14 \pm 32.44$ & $11.00 \pm 2.57$ & $38.00 \pm 10.13$ \\
\hline Grooming $(n)^{a, b}$ & $7.00 \pm 1.69$ & $0.00 \pm 0.00$ & $5.00 \pm 1.09$ \\
\hline $\operatorname{Grooming}(\mathrm{s})^{\mathrm{a}, \mathrm{b}}$ & $37.57 \pm 13.07$ & $0.00 \pm 0.00$ & $19.57 \pm 6.59$ \\
\hline Fight $(n)^{a, b}$ & $1.57 \pm 0.57$ & $10.14 \pm 1.79$ & $1.71 \pm 0.64$ \\
\hline Fight $(s)^{a, b}$ & $1.57 \pm 0.57$ & $12.43 \pm 2.66$ & $2.00 \pm 0.90$ \\
\hline $\operatorname{Aggressive}(\mathrm{n})^{\mathrm{a}, \mathrm{b}}$ & $1.43 \pm 0.48$ & $3.29 \pm 0.57$ & $0.43 \pm 0.43$ \\
\hline Aggressive $(\mathrm{s})^{\mathrm{b}}$ & $3.71 \pm 1.39$ & $7.71 \pm 1.35$ & $0.71 \pm 0.71$ \\
\hline $\operatorname{Biting}(n)^{a, b}$ & $0.00 \pm 0.00$ & $7.57 \pm 1.48$ & $0.14 \pm 0.14$ \\
\hline $\operatorname{Biting}(s)^{a, b}$ & $0.00 \pm 0.00$ & $9.86 \pm 2.04$ & $0.14 \pm 0.14$ \\
\hline
\end{tabular}

Data are presented as the means \pm SEM. 'Grooming' denotes grooming partners; 'Aggressive' denotes aggressive grooming. n, number of times behavior was noted; s, duration measured in seconds; NS, non-stressed group; PNS, prenatally-stressed group; VF, oral administration of Valeriana fauriei to the prenatally-stressed group; ${ }^{a} \mathrm{Comparison}$ between NS and PNS, $\mathrm{p}<0.05$; ${ }^{\mathrm{b}}$ comparison between PNS and VF, $\mathrm{p}<0.05$.

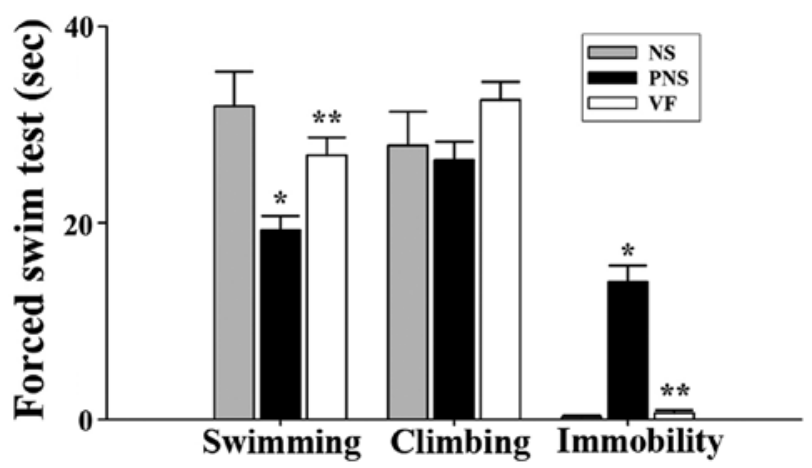

Figure 2. Behavioral responses in the forced swim test. ${ }^{*} \mathrm{p}<0.05$, comparison between NS and PNS groups; ${ }^{* *} \mathrm{p}<0.05$, comparison between PNS and VF groups. The rats in the PNS group exhibited decreased swimming ability and increased immobility. Data are presented as the means \pm SEM. NS, offspring of non-stressed rats; PNS, offspring of prenatally-stressed rats; VF, Valeriana fauriei-treated offspring.

group decreased and was close to the control level $(\mathrm{p}<0.05$, Fig. 3).

Western blot analysis and immunohistochemistry. To examine the PNS-induced downregulation of several neurodevelopmental proteins, namely Lasp1, Dpys12, Dlg4 and the Nefm proteins, we performed western blot analysis (Fig. 4) and immunohistochemical analyses (Figs. 5-7) of the prefrontal cortex areas of the brains of rats in the NS, PNS and VF-treated groups. Western blot analysis revealed that the quantities of these four proteins in the prefrontal cortex were significantly lower in the PNS group than in the NS group ( $p<0.05$; Fig. 4). 


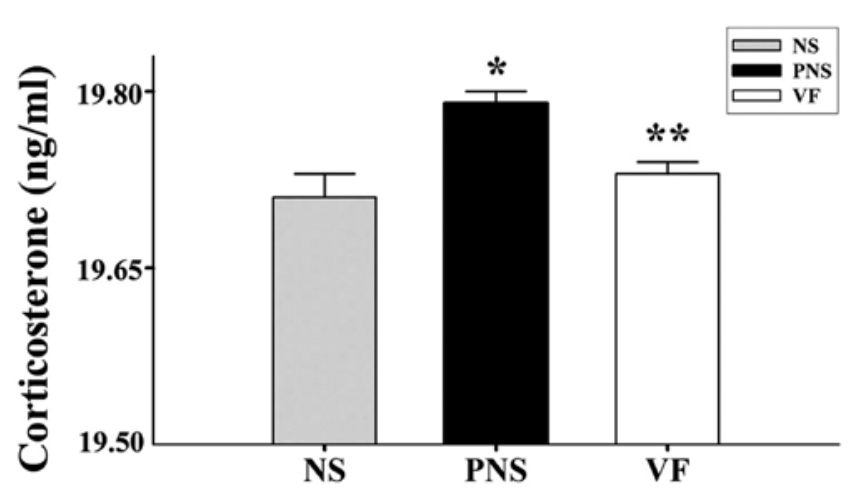

Figure 3. Corticosterone levels in adult male rats. These were analyzed on postnatal day 59 in the male offspring of rats exposed to stress during gestation or male rats derived from mothers that were not stressed during gestation. Corticosterone secretion was induced by holding the rats in restraint tubes for 60 min. ${ }^{*} \mathrm{p}<0.05$, comparison between NS and PNS groups; ${ }^{* *} \mathrm{p}<0.05$, comparison between PNS and VF groups. Corticosterone levels were increased in the PNS group and decreased in the VF treatment group. Data are presented as the means \pm SEM. NS, offspring of non-stressed rats; PNS, offspring of rats which were prenatally stressed; VF, Valeriana fauriei-treated offspring from prenatally-stressed rats.

These changes were reversed by VF treatment ( $<<0.05$; Fig. 4). Dpys12, Dlg4 and Nefm were differentially expressed, as shown in the immunofluorescent-stained brain images of the NS, PNS and VF-treated groups as well as in the immunohistochemical staining intensity values ( $\mathrm{p}<0.05$, Figs. 5-7).

\section{Discussion}

In this study, we performed behavioral tests as well as protein expression analyses in a rat model of PNS in order to examine the potential preventive effects of VF on the pathophysiology of stress-related psychiatric disorders, such as depression and schizophrenia, according to neurodevelopmental theory (50).
Certain preclinical studies have focused on the antidepressant-like effect of the Valeriana species (45), and the alterations in cerebral $\mathrm{Na}^{+} / \mathrm{K}^{+}$-ATPase activity caused by Valerian species in other psychiatric disorders $(46,47)$. Additionally, valerian root extract has been proven to exert neuroprotective effects in several neurodegenerative diseases, such as Parkinson's and Alzheimer's disease (13-15). It has also been reported that the effects of valerian and its active component valerenic acid are closely related to the GABA system $(11,48)$. GABA-induced depolarization activates cAMP response element-binding signaling, which promotes neuronal survival by activating downstream survival genes (49).

Experimental manipulation of the PNS model interrupts early brain and central nervous system development, thus inducing significant neurodevelopmental changes, increasing maternal stress hormones, and altering responses to prenatal stressors $(23,50)$. Impaired social behavior and interaction was observed in prepubertal rats at 56 days of age as well as in young adult rats and all these rats were the offspring of PNS-exposed rats (56). One of the first clinical signs associated with human schizophrenia is social withdrawal during adolescence (51-53). The emergence of social withdrawal in adolescent rats in the PNS group appears to be consistent with the literature on clinical schizophrenia and further supports the theory that this model is relevant to studies of the schizophrenia phenotype. This diminution of social behavior and interaction may reflect the increased anxiety experienced by the offspring of the prenatally-stressed rats (24). In the present study, PNS-induced decreases in non-aggressive behavior and increases in aggressive behavior returned towards normal levels following VF treatment. Additionally, certain behavioral patterns noted in the FST and OFT, which assess depressive behavior, were recovered following VF treatment.

We investigated sensorimotor gating, as reflected by PPI $(54,55)$. This gating has been reported to be disrupted in patients with schizophrenia (56), but de Bruin et al (57) demonstrated that these forms of gating are independent
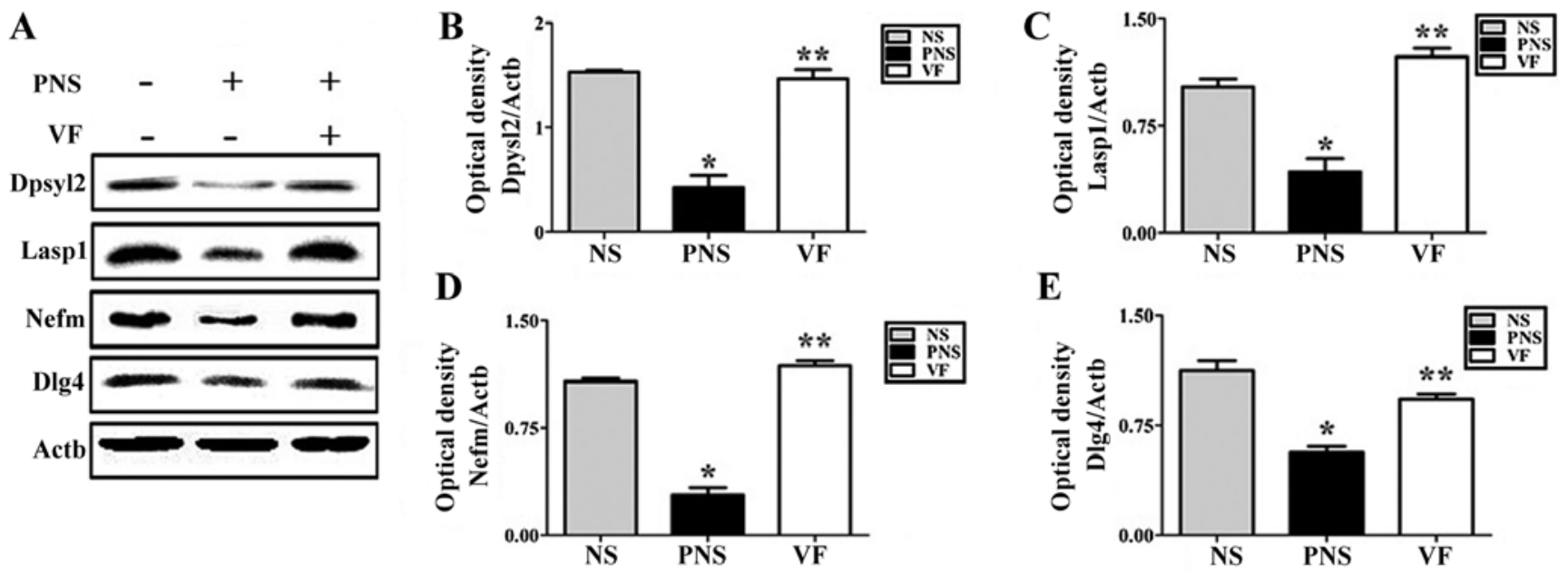

Figure 4. Western blot analysis of dihydropyrimidinase-like 2 (Dpys12), LIM and SH3 protein 1 (Lasp1), neurofilament M (Nefm) and discs, large homolog 4 (Dlg4) expression in the brains of the offspring of rats exposed to prenatal stress (PNS). (A) Dpys12, Lasp1, Nefm and Dlg4 expression was detected by western blot analysis; Actb was used as the internal control. Rats in the PNS group exhibited decreased Dpys12, Lasp1, Nefm and Dlg4 expression in the prefrontal cortex. Decreased Dpys12, Lasp1, Nefm and Dlg4 expression recovered after Valeriana fauriei treatment. Quantitative analysis of (B) Dpys12 expression, (C) Lasp1 expression, (D) Nefm expression and (E) Dlg4 expression. The data in the graphs represent the means $\pm \mathrm{SEM}$. ${ }^{*} \mathrm{P}<0.05$, comparison between NS and PNS groups; ${ }^{* *} \mathrm{P}<0.05$, comparison between PNS and VF groups. NS, offspring of non-stressed rats; PNS, offspring of prenatally-stressed rats; VF, Valeriana fauriei-treated offspring. 



Figure 5. Immunohistochemical analysis of dihydropyrimidinase-like 2 (Dpys12) expression in the brains of the offspring of rats exposed to PNS. (A) Confocal microscopic images showing immunofluorescent staining for Dpys12 (anti-Dpys12, red, Cy3) with NeuN in the prefrontal cortex. Fluorescent staining revealed a decrease in Dpysl2 expression in the PNS group. Scale bar, $50 \mu \mathrm{m}$. (B) Quantitative analysis of Dpysl2 expression. The data in the graphs represent the means \pm SEM. ${ }^{*} \mathrm{P}<0.05$, comparison between NS and PNS groups; ** P $<0.05$, comparison between PNS and VF groups. NS, offspring of non-stressed rats; PNS, offspring of prenatally-stressed rats; VF, Valeriana fauriei-treated offspring.


Figure 6. Immunohistochemical analysis of neurofilament M (Nefm) expression in the brains of the offspring of rats exposed to PNS. (A) Confocal microscopic images showing immunofluorescent staining for Nefm (anti-Nefm, green, Alexa Fluor 488) with DAPI (blue) in the prefrontal cortex. Fluorescent staining revealed decreased Nefm expression in the PNS group. Scale bar, $50 \mu \mathrm{m}$. (B) Quantitative analysis of Nefm expression. The data in the graphs represent the means \pm SEM. ${ }^{*} \mathrm{P}<0.05$, comparison between NS and PNS groups; ${ }^{* *} \mathrm{P}<0.05$, comparison between PNS and VF groups. NS, offspring of non-stressed rats; PNS, offspring of prenatally-stressed rats; VF, Valeriana fauriei-treated offspring.
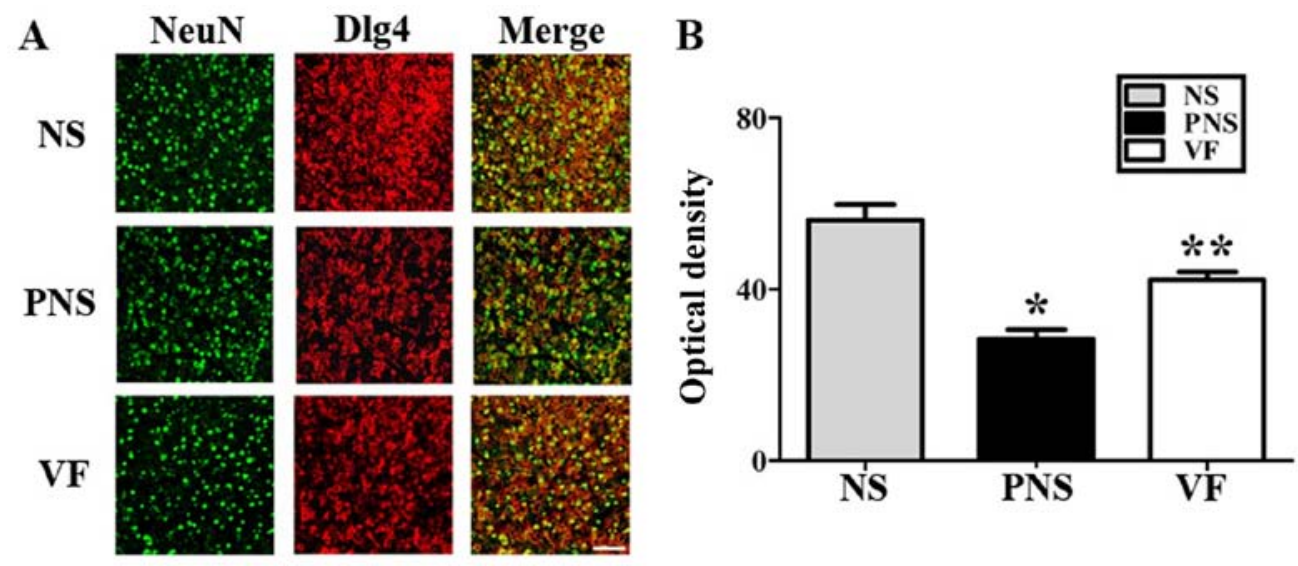

Figure 7. Immunohistochemical analysis of discs, large homolog 4 (Dlg4) expression in the brains of the offspring of rats exposed to PNS. (A) Confocal microscopic images showing immunofluorescent staining for Dlg4 (anti-Dlg4, red, Cy3) with NeuN in the prefrontal cortex. Fluorescent staining revealed decreased Dlg4 expression in the PNS group. Scale bar, $50 \mu \mathrm{m}$. (B) Quantitative analysis of Nefm expression. The data in the graphs represent the means \pm SEM. ${ }^{*} \mathrm{P}<0.05$, comparison between NS and PNS groups; * $\mathrm{P}<0.05$ comparison between PNS and VF groups. NS, offspring of non-stressed rats; PNS, offspring of prenatally-stressed rats; VF, Valeriana fauriei-treated offspring. 
central nervous system (CNS) phenomena. Previous studies have noted PPI deficits in a neonatal rat model of ventral hippocampal lesions (58), heterozygous reeler mice (59), rats reared in isolation (60), and rats which received a prenatal immune challenge (61), and a mouse model of maternal influenza (62). However, Lehmann et al failed to generate PPI deficits by repeatedly stressing Wistar female rats during the final week of gestation (63). We suggest that several factors including the rat strain $(64,65)$ and the homotypic stress paradigm used by Lehmann et al (63) contributed to the different outcomes. We report in the present study that repeated exposure to various stressors disrupted the gating, as reflected by deficits in PPI, and this was affected by VF treatment.

The decrease in the expression of neurofilament and Dpysl2 proteins in the offspring of rats with PNS was affected by VF treatment. Our previous studies have shown that Dpysl2 and neurofilament protein levels decreased in the offspring of rats exposed to PNS $(36,37)$. Furthermore, according to our unpublished data, Lasp1 protein was also downregulated in the PNS group. To confirm these results and to verify the effect of $\mathrm{VF}$ on the expression of the proteins, in the present study we examined protein expression in the brains of the offspring of the VF-treated group. We detected the decreased expression of a postsynaptic protein associated with the development of the dendritic spine called Dlg4 (also known as PSD95) for the first time, to the best of our knowledge, in the rat model of PNS. Thus, we conclude that PNS induced decreases in the expression of several neurodevelopmental proteins and the expression levels were increased following VF treatment. These changes in protein expression may affect brain development and may have influenced the behavioral changes in the offpsring of the prenatally-stressed rats.

Our results illustrate the beneficial effect which VF exerts, and we suggest that it would be useful in the treatment of psychiatric disorders such as schizophrenia. However, further research using cellular and animal model systems with a single extract component is necessary in order to characterize the potential pharmacological functions of VF in models of schizophrenia and depression-like behavior.

\section{Acknowledgements}

The present study was performed with the support of the 'Cooperative Research Program for Agriculture Science and Technology Development (project no. PJ0115822016)' of the Rural Development Administration, Korea.

\section{References}

1. Stevinson C and Ernst E: Valerian for insomnia: a systematic review of randomized clinical trials. Sleep Med 1: 91-99, 2000.

2. Circosta C, De Pasquale R, Samperi S, Pino A and Occhiuto F: Biological and analytical characterization of two extracts from Valeriana officinalis. J Ethnopharmacol 112: 361-367, 2007.

3. Kim JS, Ahn JD and Cho SI: Effects of Valerianae Radix et Rhizoma extract on psychological stress in mice. Pharmacogn Mag 11: 381-388, 2015.

4. Barton DL, Atherton PJ, Bauer BA, Moore DF Jr, Mattar BI, Lavasseur BI, Rowland KM Jr, Zon RT, Lelindqwister NA, Nagargoje GG, et al: The use of Valeriana officinalis (Valerian) in improving sleep in patients who are undergoing treatment for cancer: a phase III randomized, placebo-controlled, double-blind study (NCCTG Trial, N01C5). J Support Oncol 9: 24-31, 2011.
5. Rezvani ME, Roohbakhsh A, Allahtavakoli $M$ and Shamsizadeh A: Anticonvulsant effect of aqueous extract of Valeriana officinalis in amygdala-kindled rats: possible involvement of adenosine. J Ethnopharmacol 127: 313-318, 2010.

6. Murphy K, Kubin ZJ, Shepherd JN and Ettinger RH: Valeriana officinalis root extracts have potent anxiolytic effects in laboratory rats. Phytomedicine 17: 674-678, 2010.

7. Wang PC, Ran XH, Chen R, Luo HR, Liu YQ, Zhou J and Zhao YX: Germacrane-type sesquiterpenoids from the roots of Valeriana officinalis var. latifolia. J Nat Prod 73: 1563-1567, 2010.

8. Letchamo W, Ward W, Heard B and Heard D: Essential oil of Valeriana officinalis L. cultivars and their antimicrobial activity as influenced by harvesting time under commercial organic cultivation. J Agric Food Chem 52: 3915-3919, 2004.

9. Leuschner J, Müller J and Rudmann M: Characterisation of the central nervous depressant activity of a commercially available valerian root extract. Arzneimittelforschung 43: 638-641, 1993.

10. Hadley S and Petry JJ: Valerian. Am Fam Physician 67: 1755-1758, 2003

11. Yuan CS, Mehendale S, Xiao Y, Aung HH, Xie JT and Ang-Lee MK: The gamma-aminobutyric acidergic effects of valerian and valerenic acid on rat brainstem neuronal activity. Anesth Analg 98: 353-358, 2004.

12. Sudati JH, Fachinetto R, Pereira RP, Boligon AA, Athayde ML, Soares FA, de Vargas Barbosa NB and Rocha JB: In vitro antioxidant activity of Valeriana officinalis against different neurotoxic agents. Neurochem Res 34: 1372-1379, 2009.

13. de Oliveria DM, Barreto G, De Andrade DVG, Saraceno E, Aon-Bertolino L, Capani F, Dos Santos El Bachá R and Giraldez LD: Cytoprotective effect of Valeriana officinalis extract on an in vitro experimental model of Parkinson disease. Neurochem Res 34: 215-220, 2009.

14. Malva JO, Santos S and Macedo T: Neuroprotective properties of Valeriana officinalis extracts. Neurotox Res 6: 131-140, 2004.

15. Pereira RP, Fachinetto R, de Souza Prestes A, Wagner C, Sudati JH, Boligon AA, Athayde ML, Morsch VM and Rocha JBT: Valeriana officinalis ameliorates vacuous chewing movements induced by reserpine in rats. J Neural Transm Vienna 118: 1547-1557, 2011.

16. Brown AS, van Os J, Driessens C, Hoek HW and Susser ES: Further evidence of relation between prenatal famine and major affective disorder. Am J Psychiatry 157: 190-195, 2000.

17. Sullivan PF: The genetics of schizophrenia. PLoS Med 2: e212, 2005.

18. Huttunen MO and Niskanen P: Prenatal loss of father and psychiatric disorders. Arch Gen Psychiatry 35: 429-431, 1978.

19. King M, Nazroo J, Weich S, McKenzie K, Bhui K, Karlsen S, Stansfeld S, Tyrer P, Blanchard M, Lloyd K, et al: Psychotic symptoms in the general population of England - a comparison of ethnic groups (The EMPIRIC study). Soc Psychiatry Psychiatr Epidemiol 40: 375-381, 2005.

20. King S, Laplante D and Joober R: Understanding putative risk factors for schizophrenia: retrospective and prospective studies. J Psychiatry Neurosci 30: 342-348, 2005.

21. Lim C, Chong SA and Keefe R: Psychosocial factors in the neurobiology of schizophrenia: a selective review. Ann Acad Med Singapore 38: 402-406, 2009.

22. Imamura Y, Nakane Y, Ohta Y and Kondo H: Lifetime prevalence of schizophrenia among individuals prenatally exposed to atomic bomb radiation in Nagasaki City. Acta Psychiatr Scand 100: 344-349, 1999.

23. Meyer U and Feldon J: Epidemiology-driven neurodevelopmental animal models of schizophrenia. Prog Neurobiol 90: 285-326, 2010.

24. Weinstock M: The long-term behavioural consequences of prenatal stress. Neurosci Biobehav Rev 32: 1073-1086, 2008.

25. Seckl JR: Prenatal glucocorticoids and long-term programming. Eur J Endocrinol 151 (Suppl 3): U49-U62, 2004.

26. de Kloet ER, Sibug RM, Helmerhorst FM and Schmidt MV: Stress, genes and the mechanism of programming the brain for later life. Neurosci Biobehav Rev 29: 271-281, 2005.

27. Beydoun H and Saftlas AF: Physical and mental health outcomes of prenatal maternal stress in human and animal studies: a review of recent evidence. Paediatr Perinat Epidemiol 22: 438-466, 2008.

28. Lee PR, Brady DL, Shapiro RA, Dorsa DM and Koenig JI: Prenatal stress generates deficits in rat social behavior: reversal by oxytocin. Brain Res 1156: 152-167, 2007. 
29. Kinnunen AK, Koenig JI and Bilbe G: Repeated variable prenatal stress alters pre- and postsynaptic gene expression in the rat frontal pole. J Neurochem 86: 736-748, 2003.

30. Koenig JI,Elmer GI, Shepard PD,LeePR, Mayo C,Joy B,HercherE and Brady DL: Stress during gestation produces alterations in adult rat behavior: relevance to schizophrenia. Soc Neurosci abs. 495.6 http://www.sfn.org/Annual-Meeting/Past-and-Future-AnnualMeetings/Abstract-Archive/Abstract-Archive-Detail?AbsYear $=2002 \& A b s I D=7512$.

31. Koenig JI, Elmer GI, Shepard PD, Lee PR, Mayo C, Joy B Hercher E and Brady DL: Prenatal exposure to a repeated variable stress paradigm elicits behavioral and neuroendocrinological changes in the adult offspring: potential relevance to schizophrenia. Behav Brain Res 156: 251-261, 2005.

32. Lemaire V, Koehl M, Le Moal M and Abrous DN: Prenatal stress produces learning deficits associated with an inhibition of neurogenesis in the hippocampus. Proc Natl Acad Sci USA 97: 11032-11037, 2000.

33. Martínez-Téllez RI, Hernández-Torres E, Gamboa C and Flores G: Prenatal stress alters spine density and dendritic length of nucleus accumbens and hippocampus neurons in rat offspring. Synapse 63: 794-804, 2009.

34. Van den Hove DL, Kenis G, Brass A, Opstelten R, Rutten BP, Bruschettini M, Blanco CE, Lesch KP, Steinbusch HW and Prickaerts J: Vulnerability versus resilience to prenatal stress in male and female rats; implications from gene expression profiles in the hippocampus and frontal cortex. Eur Neuropsychopharmacol 23: 1226-1246, 2013.

35. Mairesse J, Vercoutter-Edouart AS, Marrocco J, Zuena AR Giovine A, Nicoletti F, Michalski JC, Maccari S and Morley-Fletcher S: Proteomic characterization in the hippocampus of prenatally stressed rats. J Proteomics 75: 1764-1770, 2012 .

36. Lee H, Joo J, Nah SS, Kim JW, Kim HK, Kwon JT, Lee HY, Kim YO and Kim HJ: Changes in Dpysl2 expression are associated with prenatally stressed rat offspring and susceptibility to schizophrenia in humans. Int J Mol Med 35: 1574-1586, 2015.

37. Kim YO, Lee HY, Won H, Nah SS, Lee HY, Kim HK, Kwon JT and Kim HJ: Influence of Panax ginseng on the offspring of adult rats exposed to prenatal stress. Int J Mol Med 35: 103-109, 2015.

38. Dulawa SC, Holick KA, Gundersen B and Hen R: Effects of chronic fluoxetine in animal models of anxiety and depression. Neuropsychopharmacology 29: 1321-1330, 2004.

39. Schroeder M, Sultany T and Weller A: Prenatal stress effects on emotion regulation differ by genotype and sex in prepubertal rats. Dev Psychobiol 55: 176-192, 2013.

40. Becker A, Peters B, Schroeder H, Mann T, Huether G and Grecksch G: Ketamine-induced changes in rat behaviour: a possible animal model of schizophrenia. Prog Neuropsychopharmacol Biol Psychiatry 27: 687-700, 2003.

41. Koenig JI, Elmer GI, Shepard PD, Lee PR, Mayo C, Joy B, Hercher E and Brady DL: Prenatal exposure to a repeated variable stress paradigm elicits behavioral and neuroendocrinological changes in the adult offspring: potential relevance to schizophrenia. Behav Brain Res 156: 251-261, 2005.

42. Chang CH, Hsiao YH, Chen YW, Yu YJ and Gean PW: Social isolation-induced increase in NMDA receptors in the hippocampus exacerbates emotional dysregulation in mice. Hippocampus 25 : 474-485, 2015.

43. Morley-Fletcher S, Darnaudery M, Koehl M, Casolini P, Van Reeth $\mathrm{O}$ and Maccari S: Prenatal stress in rats predicts immobility behavior in the forced swim test. Effects of a chronic treatment with tianeptine. Brain Res 989: 246-251, 2003.

44. Joo J, Lee S, Nah SS, Kim YO, Kim DS, Shim SH, Hwangbo Y, Kim HK, Kwon JT, Kim JW, et al: Lasp1 is down-regulated in NMDA receptor antagonist-treated mice and implicated in human schizophrenia susceptibility. J Psychiatr Res 47: 105-112, 2013.

45. Liu XG, Gao PY, Wang GS, Song SJ, Li LZ, Li X, Yao XS and Zhang ZX: In vivo antidepressant activity of sesquiterpenes from the roots of Valeriana fauriei Briq. Fitoterapia 83: 599-603, 2012.
46. Ortiz JG, Nieves-Natal J and Chavez P: Effects of Valeriana officinalis extracts on $[3 \mathrm{H}]$ flunitrazepam binding, synaptosomal $[3 \mathrm{H}]$ GABA uptake, and hippocampal [3H]GABA release. Neurochem Res 24:1373-1378, 1999.

47. Patočka and Jakl J: Biomedically relevant chemical constituents of Valeriana officinalis. J Appl Biomed 8: 11-18, 2010

48. Santos MS, Ferreira F, Cunha AP, Carvalho AP and Macedo T: An aqueous extract of valerian influences the transport of GABA in synaptosomes. Planta Med 60: 278-279, 1994.

49. Merz K, Herold S and Lie DC: CREB in adult neurogenesis - master and partner in the development of adult-born neurons? Eur J Neurosci 33: 1078-1086, 2011.

50. Charil A, Laplante DP, Vaillancourt C and King S: Prenatal stress and brain development. Brain Res Brain Res Rev 65: 56-79, 2010.

51. Kelley ME, Gilbertson M, Mouton A and van Kammen DP. Deterioration in premorbid functioning in schizophrenia: a developmental model of negative symptoms in drug-free patients. Am J Psychiatry 149: 1543-1548, 1992.

52. Møller P and Husby R: The initial prodrome in schizophrenia: searching for naturalistic core dimensions of experience and behavior. Schizophr Bull 26: 217-232, 2000.

53. Cornblatt BA: The New York high risk project to the Hillside recognition and prevention (RAP) program. Am J Med Genet 114: 956-966, 2002.

54. Geyer MA, Krebs-Thomson K, Braff DL and Swerdlow NR Pharmacological studies of prepulse inhibition models of sensorimotor gating deficits in schizophrenia: a decade in review. Psychopharmacology (Berl) 156: 117-154, 2001.

55. Swerdlow NR and Geyer MA: Using an animal model of deficient sensorimotor gating to study the pathophysiology and new treatments of schizophrenia. Schizophr Bull 24: 285-301, 1998.

56. Braff DL: Information processing and attention dysfunctions in schizophrenia. Schizophr Bull 19: 233-259, 1993.

57. de Bruin NM, van Luijtelaar EL, Cools AR and Ellenbroek BA: Auditory information processing in rat genotypes with different dopaminergic properties. Psychopharmacology (Berl) 156: 352-359, 2001.

58. Lipska BK and Weinberger DR: To model a psychiatric disorder in animals: schizophrenia as a reality test. Neuropsychopharmacology 23: 223-239, 2000.

59. Tueting P, Costa E, Dwivedi Y, Guidotti A, Impagnatiello F, Manev R and Pesold C: The phenotypic characteristics of heterozygous reeler mouse. Neuroreport 10: 1329-1334, 1999.

60. Day-Wilson KM, Jones DN, Southam E, Cilia J and Totterdell S: Medial prefrontal cortex volume loss in rats with isolation rearing-induced deficits in prepulse inhibition of acoustic startle. Neuroscience 141: 1113-1121, 2006.

61. Borrell J, Vela JM, Arévalo-Martin A, Molina-Holgado E and Guaza C: Prenatal immune challenge disrupts sensorimotor gating in adult rats. Implications for the etiopathogenesis of schizophrenia. Neuropsychopharmacology 26: 204-215, 2002.

62. Shi L, Fatemi SH, Sidwell RW and Patterson PH: Maternal influenza infection causes marked behavioral and pharmacological changes in the offspring. J Neurosci 23: 297-302, 2003.

63. Lehmann J, Stöhr T and Feldon J: Long-term effects of prenatal stress experiences and postnatal maternal separation on emotionality and attentional processes. Behav Brain Res 107: $133-144,2000$

64. Faraday MM, O'Donoghue VA and Grunberg NE: Effects of nicotine and stress on startle amplitude and sensory gating depend on rat strain and sex. Pharmacol Biochem Behav 62: 273-284, 1999.

65. Stöhr T, Schulte Wermeling D, Szuran T, Pliska V, Domeney A, Welzl H, Weiner I and Feldon J: Differential effects of prenatal stress in two inbred strains of rats. Pharmacol Biochem Behav 59: 799-805, 1998. 